\title{
FAST MEASUREMENT OF SOLUBLE SOLID CONTENT IN MANGO BASED ON VISIBLE AND INFRARED SPECTROSCOPY TECHNIQUE
}

\author{
Jiajia Yu, Yong $\mathrm{He}^{*}$ \\ College of Biosystems Engineering and Food Science, Zhejiang University, 268 Kaixuan \\ Road, Hangzhou, 310029, China \\ * Corresponding author, Address: College of Biosystems Engineering and Food \\ Science,Zhejiang University, 268 Kaixuan Road, Hangzhou, 310029, China, Tel: +86- \\ 571-86971143, Fax: +86-571-86971143,Email: yhe@zju.edu.cn
}

\begin{abstract}
Mango is a kind of popular tropical fruit, and the soluble solid content is an important in this study visible and short-wave near-infrared spectroscopy (VIS/SWNIR) technique was applied. For sake of investigating the feasibility of using VIS/SWNIR spectroscopy to measure the soluble solid content in mango, and validating the performance of selected sensitive bands, for the calibration set was formed by 135 mango samples, while the remaining 45 mango samples for the prediction set. The combination of partial least squares and backpropagation artificial neural networks (PLS-BP) was used to calculate the prediction model based on raw spectrum data. Based on PLS-BP, the determination coefficient for prediction $(\mathrm{Rp})$ was 0.757 and root mean square error for prediction (RMSEP) was 0.865. It is concluded that infrared spectroscopy technique can quantitative the soluble solid content in mango, and the process is simple and easy to operate. Compared with the Partial least squares (PLS) result, the performance of PLS-BP is better.
\end{abstract}

Key words: Mango, soluble solid content, PLS-BP, near-infrared spectroscopy

\section{INTRODUCTION}

Mango is a kind of popular tropical fruit. It is rich in organic acid and carotene, and it is benefit to people's health. Nowadays, a good tasting and high nutrition is the critical factors to commercial benefits. As a result, it is

Please use the following format when citing this chapter:

Yu, J. and He, Y., 2009, in IFIP International Federation for Information Processing, Volume 293, Computer and Computing Technologies in Agriculture II, Volume 1, eds. D. Li, Z. Chunjiang, (Boston: Springer), pp. 89-95. 
urgent to find a better way to determine the quality to ensure farmers' interests. However, most techniques available for soluble solid content analysis are time consuming, destructive and costly, and require professional operations. Therefore, it is much-needed for the development of a reliable, non-destructive method.

Recently, the visible and short-wave near-infrared spectroscopy (VIS/SWNIR) technique (wavelengths range from 400 to $1075 \mathrm{~nm}$ in this study), has received interest because of its non-destructive analyses for biological and biomedical materials. Because of its high transmittance and fast responses, it is suitable as an excellent detector, In the VIS/SWNIR region, there are several advantages such as the signal exploitation is reliable, the measurement time is low (J. B. Reeves et al., 1994) and the effective of intense water bands in NIR region can be diminished (S. Sasic et al.,2001).

For the recently quantitative analysis, Saona et al. determined individual sugars in fruit juices using FT-NIR and multivariate techniques (Saona et al.,2001), Liu et al. used visible and near infrared spectroscopy to assess soluble solids content and $\mathrm{pH}$ of rice wine.( Liu, F. et al.,2007)

Since mango is rich in organic acid and carotene, and the organic acid and carotene can influence the soluble solid content (Shao, Y. N.et al.,2007), which leads to highly overlapped peaks and regardless of bandwidth in infrared spectroscopy. At the same time there is much noise and other unrelated information arises from overtones and combinations of such vibrations, rendering them much more difficult to interpret. For sake of finding the relevant quantitative information, especially the nonlinear one, it is necessary to use the suitable and effective chemometrics methods. Propagation artificial neural networks in this study was used.

The aim of this study was to explore the feasibility of using VIS/SWNIR spectroscopy to discriminate different brands of mango. A nonlinear mathematical method for rapid, nondestructive identification of lubricating brands was developed by BP-ANNs. In order to validate the performance of selected effective wavelengths of mango, loading weights in Partial Least Squres (PLS) were analyzed.

\section{MATERIALS AND METHODS}

\subsection{Sample preparation}

180 mango samples were purchased in local market. They were stored in the lab for 24 hours with a constant temperature of $25 \pm 1{ }^{\circ} \mathrm{C}$ to equalize the temperature. 135 mango samples were randomly selected for the calibration set, while the remaining 45 samples for the prediction set. 


\subsection{Spectra measurement}

For each sample, reflectance spectra were scanned by a handheld FieldSpec Pro FR (325-1075 nm)/A110070, Trademarks of Analytical Spectral Devices, Inc. (Analytical Spectral Devices, Boulder, USA) for three times each. The light source consists of a Lowell pro-lam interior light source assemble/128930 with Lowell prolam $14.5 \mathrm{~V} \mathrm{Bulb/128690}$ tungsten halogen bulb that could be used both in visible and near infrared region. The field-of-view (FOV) of the spectroradiometer is $10^{\circ}$. The spectroradiometer was placed at a height of approximately $250 \mathrm{~mm}$ and $45^{\circ}$ angle away from the center of sample. The light source was placed at a height of approximately $150 \mathrm{~mm} 45^{\circ}$ angle away from the sample. The spectrum of each sample was the average of 30 successive scans with $1.5 \mathrm{~nm}$ intervals. Three spectra were collected for each sample and the average spectrum of these two measurements was used in the later analysis. All spectral data were stored in a computer and processed using the RS3 software for Windows (Analytical Spectral Devices, Boulder, USA) designed with a Graphical User Interface.

\subsection{Spectral data pretreatment}

The reflectance spectra were firstly transformed into ASCII format by using the ASD ViewSpecPro software (Analytical Spectral Devices, Boulder, USA). Then three spectra for each sample were averaged into one spectrum and transformed by $\log (1 / \mathrm{T})$ into absorbance spectrum. The pretreatments were implemented by "The Unscrambler V 9.7" (CAMO PROCESS AS, OSLO, Norway). Because of the high noise at the beginning of the spectrum, 421-1075 nm was used in this study. After some trial computations, the optimal smoothing way of moving average with 3 segments was applied to decrease the noise. Standard normal variate (SNV) was applied for light scatter correction and reducing the changes of light path length, but it was showed the raw spectrum data's result was closed to the pretreated data. In order to reduce the processing time, in this study the raw spectrum data was chosen.

\subsection{Backpropagation artificial neural networks}

Backpropagation artificial neural networks have one or more hidden layers nodes besides input node and output node, and there is no coupling in the same hidden layer (M. R. Widyanto,et al.2005). The input signals can be passed through hidden layer node from the input node to the output node. The output of on layer becomes the input to the following layer. The transfer function of node takes the form:

$$
f(x)=1 /\left(1+e^{-Q / X}\right)
$$


In the function, $\mathrm{Q}$ is the parameter of function, in this study Sigmoid was used.

Propagation artificial neural networks is a kind of multi-storey network used Widrow-Hoff learning algorithm and nonlinear differentiable functions. Gradient descent algorithm is used in a typical BP network, which is Widrow-Hoff algorithm requirements (Aleksandra Vuckovic,et al.2002). Backpropagation is referred to this kind of algorithm. This recursive algorithm can be rewritten as:

$$
x k+1=x k-a k \bullet g k
$$

Here $x k$ is the current weight and bias vector, $g k$ is the current gradient, $a k$ is the learning rate.

\subsection{Reference method for SSC}

The reference value of SSC was measured by an Abbebenchtop refractometer (Model: WAY-2S, Shanghai Precision \& Scientific Instrument Co. Ltd., Shanghai, China). The refractive index accuracy is \pm 0.0002 and the Brix (\%) range is $0-95 \%$ with temperature correction.

\section{RESULT AND DISCUSSION}

\subsection{Spectral Features of mango}

Fig 1. shows the transmission's spectrum of mango. Because there were a lot of noises between $350 \mathrm{~nm}$ and $400 \mathrm{~nm}$, in this study $400 \mathrm{~nm}-1075 \mathrm{~nm}$ was used.

As seen from the picture, in visible spectrum, the absorption in $400 \sim 550$ $\mathrm{nm}$ is greater than $550 \sim 770 \mathrm{~nm}$ 's absorption, it is caused by the mango's yellow skin pigments and carotenoids (Manuela Zude,et al.2006). And in near infrared spectrum,there is a peak between $950 \mathrm{~nm}$ and $1075 \mathrm{~nm}$, it may be caused by hydroxy and hydrocarbyl. The skin's colour and organic compounds could influence the soluble solid content. So it could be seen that the VIS/NIR technique maybe a way to determine the soluble solid content. 


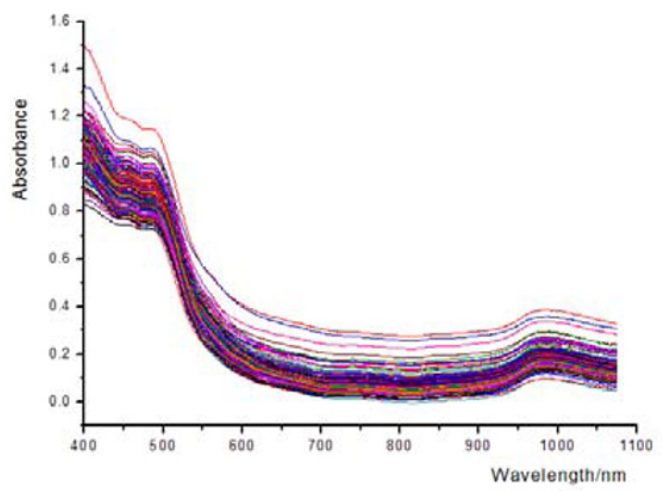

Figure 1. Whole Absorbance spectra of mango

\subsection{The soluble solid content of mango}

From the Fig. 2. and Table 1. it can finded that the reference value of SSC for mango had a high variation coefficient. It may affect the modeling results, but also benefit to find more outstanding model.

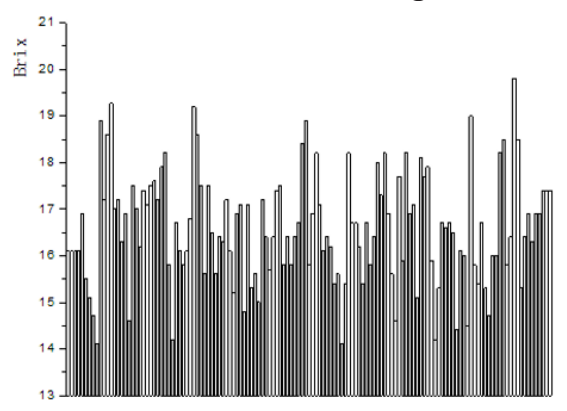

(a)

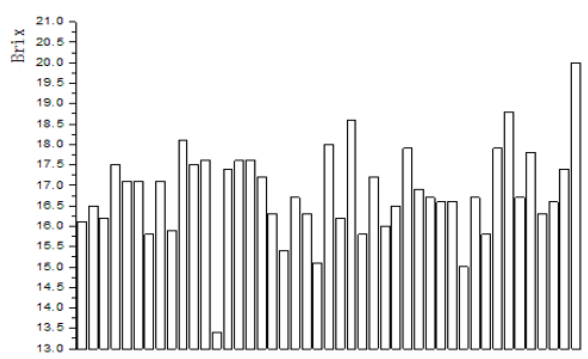

(b)

Figure 2. The SSC of calibration set (a) and prediction set(b) 
Table 1. The SSC value of mango

\begin{tabular}{lcc}
\hline \multicolumn{1}{c}{ Set } & Calibration & Prediction \\
\hline Sample number & 135 & 45 \\
max & 20.00 & 20.00 \\
min & 14.10 & 14.20 \\
mean & 16.58 & 16.83 \\
Standard deviation & 1.96 & 1.11 \\
Coefficient of variation & 11.80 & 6.62 \\
\hline
\end{tabular}

\subsection{Spectra analysis of SSC on VIS/SWNIR}

Two models were developed using same sample sets. Based on the model developed, the PLS model was applied to the all samples. Two chemometric methods, namely PLS and BP-ANNs were applied. All samples were processed by PLS before regression to get the feature input subset (LVs) for BP models.

The results of the calibration and prediction for these two separate models are shown in Table 2. BP-ANNs models did a better job with higher determination coefficient $\mathrm{R}_{\mathrm{c}}{ }^{2}$ and $\mathrm{R}_{\mathrm{P}}{ }^{2}$ and lower Root mean square error for calibration (RMSEC) and Root mean square error for prediction (RMSEP) than PLS. RSD\% and PRESS also performed better in BP models. Thus, very good determination performance for SSC in mango can be achieved by VIS/WSNIR spectra. There is relationship between SSC and VIS/SWNIR spectra.

Table 2.Results for SSC of mango from VIS/SWNIR Spectra

\begin{tabular}{cccccc}
\hline Set & Method & PCs & $\mathrm{R}_{\mathrm{c}}$ or $\mathrm{R}_{\mathrm{P}}$ & RMSEC or RMSEP & PRESS \\
\hline \multirow{2}{*}{ Calibration } & PLS & 17 & 0.902 & 0.546 & 34.890 \\
& PLS-BP & $/$ & 1.000 & 0.021 & 0.052 \\
\multirow{3}{*}{ Prediction } & PLS & 17 & 0.660 & 0.964 & 33.871 \\
& PLS-BP & $/$ & 0.757 & 0.865 & 32.645 \\
\hline
\end{tabular}

\section{CONCLUSION}

The measurement of SSC of mango could be successfully performed through visible and short-wave near-infrared spectroscopy combined with chemometric methods of PLS and BP-ANNs. The raw spectrum was used. The precision and accuracy was achieved by PLS and LS-SVM models with the $\mathrm{R}_{\mathrm{p}}$ and RMSEP were 0.660 , and 0.964 for PLS, 0.757 and 0.865 for LSSVM, respectively. RSD\% and PRESS also performed better in BP models. The results indicted that visible and short-wave NIR spectroscopy had the capability to determine the carbohydrate content in milk powder. Further 
interpretation of the input data selection, parameter optimization and results explanation would be needed in order to improve the calibration generalization and stability.

\section{ACKNOWLEDGMENT}

This study was supported by National Science and Technology Support Program (2006BAD10A09), Zhejiang Provincial Natural Science Foundation of China (Y307158) and the Teaching and Research Award Program for Outstanding Young Teachers in Higher Education Institutions of MOE, P. R. C.

\section{REFERENCES}

Aleksandra Vuckovic, Vlada Radivojevic, Andrew C. N. Chen and Dejan Popovica. Automatic recognition of alertness and drowsiness from EEG by an artificial neural network.Medical Engineering \& Physics,2002, 24(5):349-360

J. B. Reeves III. Effects of water on the spectra of model compounds, J. Near Infrared Spectrosc, 1994, 2:199-212

Liu, F., He, Y., Wang, L., \& Pan, H. M. Feasibility of the use of visible and near infrared spectroscopy to assess soluble solids content and $\mathrm{pH}$ of rice wines, Journal of Food Engineering,2007, 83(3):430-435

M. R. Widyanto, H. Novuhara, K. Kawamoto, K. Hirota, and B. Kusumoputro. Improving recognition and generalization capablility of back-propagation $\mathrm{NN}$ using a self-organized network inspired by immune algorithm (SONIA), Applied Soft Computing 2005, 6:72-84

Manuela Zude, Bernd Herold, Jean-Michel Roger. Predicting soluble solid content and firmness in apple fruit by means of laser light backscattering image analysis, Journal of Food Engineering, 2006, 77(2):254

S. Sasic, and Y. Ozaki. Short-wave near-infrared spectroscopy of biological fluids. 1. quantitative analysis of fat, protein, and lactose in raw milk by partial least-squares regression and band assignment, Anal. Chem., 2001,73:64-71

Saona, L.E.R., Fry, F. S., McLaughlin, M. A., \& Calvey, E. M. Rapid analysis of sugars in fruit juices by FT-NIR spectroscopy, Carbohydrate Research, 2001,336 (1):63-67

Shao, Y. N., \& He, Y. Nondestructive measurement of the internal quality of bayberry juice using Vis/NIR spectroscopy, Journal of Food Engineering, 2007, 9(3):1015-1019 